\title{
Understanding experiences of the self-harm of others: A qualitative exploration of the views of young people with complex mental health needs
}

\begin{abstract}
As adolescent self-harm is a growing public health concern, more research is needed to identify potential risk factors. Studies have highlighted that exposure to the self-harm of others may be a risk factor associated with engagement in self-harm. However, research investigating young people's experiences of the self-harm of others has been limited. The present qualitative study aimed to explore young people's experiences of the selfharm of others and interviewed a total of eight young people (5 females and 3 males; aged between 13 and 18 years) resident at one of two adolescent mental health inpatient units in the North of England. The interviews were analysed using interpretative phenomenological analysis and four themes were identified: 'Pre-admission exposure to self-harm', 'Exposure on the inside: An unpleasant environment', 'Helper vs helped', 'Separation from the attention seekers: competing for authenticity'. Prevention efforts to reduce the social transmission and stigma surrounding self-harm amongst young people are discussed.
\end{abstract}

\section{Keywords}

Self-harm, young people, qualitative, experiences, social transmission 


\section{Introduction}

Self-harm amongst young people is recognized an important international public health issue, although estimates of prevalence vary greatly due to numerous factors including the definition of self-harm used, how data is collated, and the age of participants (Ougrin, 2014). A systematic review of studies published between 2005 and 2011 using community samples of adolescents (aged 11-18) from a wide range of countries found that $16 \%$ reported having self-harmed at some point in their lives (Muehlenkamp, Claes, Havertape, \& Plener, 2012). Similarly, a recent survey of 13-18 year olds in England found a lifetime prevalence of self-harm 16\% (Morey, Mellon, Dailami, Verne, \& Tapp, 2016). However, a large-scale European study of school pupils undertaken in 2009-10 (Brunner, Kaess, Parzer, Fischer, \& Carli, 2014) found an average lifetime prevalence of $28 \%$, ranging from $17 \%$ to $39 \%$ across the 11 participating countries. For adolescents in psychiatric inpatient settings the prevalence of self-harm is higher still, with rates reported ranging from $40 \%$ (Nock \& Prinstein, 2004) to $72 \%$ (Thomassin, Guérin Marion, Venasse, \& Shaffer, 2017). As already noted, definitions of self-harm vary, but in this paper we use the definition most widely used in the UK, which is as an intentional act of self-injury or self-poisoning irrespective of method and motivation, whether suicidal or non-suicidal (NICE, 2011). 
Relatively little is known about why young people start self-harming and the factors associated with it (Hasking, Andrews, \& Martin, 2013). However, research suggests that social transmission, often referred to in the literature as social contagion, may play a significant role. Within both inpatient and community settings, research has shown that young people's exposure to self-harm through friends and family may be related both to the frequency of self-harm, and to its initiation (Claes, Houben, Vandereycken, Bijttebier, \& Muehlenkamp, 2010; Deliberto \& Nock, 2008; Hasking et al., 2013; Prinstein, Heilbron, Guerry, Franklin, Rancourt, Simon, \& Spirito, 2010; Taiminen, Kallio-Soukainen, Nokso-Koivisto, Kaljonen, \& Kelenius, 1998). Young people reporting self-harm have been shown to be more likely to have a parent/family member who self-harms (Hawton, Saunders \& O'Connor, 2012), have more friends who self-harm (Claes et al., 2010) and be more likely to start self-harming for social motives (Muehlenkamp, Brausch, Quigley, \& Whitlock, 2013). For example, a study of 3,757 high school students by De Leo and Heller (2004) found that self-reported exposure to others' self-harm (e.g. that of friends and family) more than tripled the risk of an individual engaging in self-harm; whilst Hawton, Rodham, Evans, and Weatherall (2002) reported that exposure to others' self-harm led to an increased risk of over five and seven times for female and male adolescents respectively.

There are competing theories for the mechanisms underlying the social transmission of self-harm, including learning, imitation, contagion, suggestion and 
assortative relating (Haw, Hawton, Niedzweidz, \& Platt, 2013), however there is currently limited empirical evidence to support these theories. Therefore, the factors behind social transmission, including whether young people are at an increased risk of self-harm due to the distress caused by exposure to the self-harm of another, or whether they learn self-harm as a coping strategy from others, warrant further exploration.

Research with a community sample of adolescents focusing on the type and strength of the relationship as a moderator of peer influence found that the most salient determinant of future self-harm is not the total number of people who self-harm the individual is exposed to, but having close friends who self-harm (Hasking et al., 2013)., This points to the need for qualitative research aimed at exploring the relational dimension of young people's journey into self-harm; how they experience and make sense of the self-harming behaviour of others, and how, if at all, this influences their own behaviour and well-being.

Searches undertaken in preparation for this study revealed no qualitative research exploring young people's experiences of the self-harm of others in community settings, however, two studies have been undertaken within inpatient settings (Crouch \& Wright, 2004; Taiminen et al., 1998). Taiminen et al's (1998) study utilised quantitative methods to investigate the prevalence of social transmission and clinical interviews to explore the psychological mechanisms behind this. The authors highlight the need for 
further qualitative exploration in this area as the results from the clinical interviews showed that participant self-harm was "influenced by interaction with other inpatients" (p. 214). However, the qualitative findings from this paper are difficult to interpret, due to the lack of methodological and analytical detail reported.

Building upon Taiminen et al.’s (1998) findings, Crouch and Wright (2004) investigated the personal and interpersonal processes involved in self-harm within an inpatient setting using Interpretative Phenomenological Analysis (IPA). The results from this study highlight a struggle for individuals to be considered 'genuine' selfharmers and not 'attention seekers', mirroring Taiminen et al.'s (1998) earlier finding that some young people who self-harm were labelled as 'fake' or a 'pretender'. Moreover, Crouch and Wright (2004) report a 'behavioural tariff'; whereby young people who self-harm were only seen as 'genuine' if a certain amount of damage was inflicted on the self-harmer; which again echoes findings in Taiminen et al. (1998) of instances of self-harm serving as "an initiation rite that strengthened group cohesion" (p.215).

Building on this small but important body of qualitative evidence, the present study used qualitative interview-based methodology to investigate further the relational context of self-harm within adolescent inpatient settings. Both Taiminen et al. (1998) and Crouch and Wright (2004) focused exclusively on exposure to the self-harm of 
peers within the inpatient environment, reflecting a concern in earlier research (e.g. Rosen and Walsh, 1989) with the idea of self-harm as a 'social contagion' with the potential to lead to 'epidemics' of self-harm. However, more recent research into selfharm in adolescent inpatients has focused on young people's exposure to self-harm prior to admission, and suggests that these experiences may be more important in the development of young people's self-harm than the self-harm they encounter as inpatients (Jarvi, Jackson, Swenson, \& Crawford, 2013). Thus the current study sought to explore adolescents' experiences of the self-harm of others (including peers, parents and other adults) both within the context of the inpatient setting but also in the wider context of their lives. In doing so, the study sought to better understand the social transmission of self-harm in young people, including the possible impact upon an adolescent's own psychological well-being and/or self-harm.

\section{Method}

Design

The aim of the study was to understand how young people in inpatient settings make sense of the self-harm of others. In keeping with this aim, the study design was based on Interpretative Phenomenological Analysis (IPA) as developed by Smith, Flowers, and Larkin (2009). IPA was chosen because it is concerned with how people make sense of significant life experiences; in this case the self-harm of others. It places 
emphasis on in-depth, idiographic exploration of data from small homogenous samples; in this case young people who all shared the experience of currently residing in a psychiatric inpatient unit. IPA is based on the double hermeneutic principle; the researcher has an explicit role in interpreting the interpretations of their participants. Thus, the researcher's own knowledge, interests and biases are acknowledged as having a potential influence on the analytic process, and are required to be reflected upon and, where necessary, 'bracketed out' of the analysis through mechanisms such as supervision and journal-keeping. The research was undertaken as part of the first author's doctorate in clinical psychology, and was supervised by the second and third authors.

Ethical and research governance approvals were obtained through a National Health Service (NHS) research ethics committee and the participating NHS trusts' R\&D departments.

Participants

Participation in the study was open to any young person aged between 13 and 18 years who was currently resident at one of two adolescent mental health inpatient units in the North of England. Young people were not able to participate in an interview before they had been admitted for a period of two weeks as it was important that their clinical care team were able to assess whether they met the inclusion/exclusion criteria 
for the study. A young person did not have to have self-harmed, in the past or present, to be a participant, in keeping with the broad focus of the study on experiences of the self-harm of others.

Young people were excluded from participating if the care team's assessment indicated that the interview process was likely to have a detrimental effect on their mental health and/or that the young person was too unwell to participate. Furthermore, young people were also excluded if the clinical team's assessment indicated cognitive or communication difficulties that would make it too difficult for the young person to engage in an interview, and/or if that the young person was unable to give informed consent.

In total, eight participants gave their consent to be interviewed; five females and three males, aged 15-17 years. All but one were recruited from the same inpatient unit. The duration of time on the inpatient ward prior to the interview varied from three weeks to seven months. It is noteworthy that the majority of participants recruited $(n=7)$ self-harmed themselves.

\section{Data collection and analysis}

Interviews were conducted by the first author, lasted between 23 and 46 minutes, and were digitally recorded. The interviews were subsequently transcribed by 
the first author and anonymised. The transcripts were analysed by the first author using IPA as described by Smith et al. (2009). The first step of IPA involves immersion in the data by reading and re-reading each transcript. Next, the researcher examined the semantic content of the transcript, making initial notes about how the participant talked about and made sense of the topic under discussion. Using these initial notations, emergent themes were then identified within the transcript. Due to the relatively large sample of participants for an IPA study super-ordinate themes were then developed for the whole sample, rather than first developing super-ordinate themes for each individual transcript as is suggested for samples smaller than six (see Smith, et al. 2009, p106107). This was done by looking for connections across the emergent themes of all participant transcripts.

In order to ensure that any interpretative biases were acknowledged and bracketed out of the final analysis, the third author (the first author's supervisor) checked the analysis at each stage from initial noting to the development of the superordinate themes. This involved reading through the analysis, highlighting any discrepancies between the first and third author's interpretations, and discussing these in supervision with the first author.

Researcher Reflexivity 
The first author's clinical work with children who self-harm highlighted the profound levels of distress experienced by children, parents, carers and professionals when exposed to such behaviour. Having been exposed to reactions of shock, distress and anger by other young people, care staff and parents, she began to question how these reactions might impact upon the young people's future engagement in self-harm. From a social constructionist position, the researcher was also interested in the social transmission of self-harm, and the ways in which social factors shape an individual's experience of self-harm.

In line with the reflexive principles of IPA, the first author held in mind how her early work experiences may have influenced her decisions in designing and executing the study and interpreting the research findings. She was particularly concerned not to give too much weight to findings that fit with her experiences and hypotheses. Therefore, it was important to have her interpretations of the young people's experiences checked by the third author.

During the interviews and whilst analysing the data, the first author kept a reflexive journal. By the nature of interviewing young people admitted to inpatient services, it is unsurprising that their accounts included upsetting stories of significant harm to self, suicidal ideation and attempts, historical trauma and severe psychological distress. Although she had been exposed to similar stories when working clinically, the cumulative effect on her of completing the interviews, transcribing the data and 
analysing their stories was overwhelming and distressing. She considered whether this cumulative effect might be similar to the distress experienced by the young people within inpatient services who are frequently exposed to the self-harming behaviour of others.

Keeping a journal also encouraged the first author to reflect on how her own position as a trainee clinical psychologist might influence the interview process, and the differences between researcher (a passive observer and listener), and clinician (providing advice and containment to people in distress). These differences in role led the researcher to reflect on her difficulties withholding emotional support. The researcher found supervision with her academic supervisor (third author) critical in unpicking these feelings.

\section{Results}

Four themes were identified from the adolescents' experiences.

\section{Theme 1: Pre-admission exposure to self-harm}

Across participant accounts, the relationship between exposure to the self-harm of others and the development of their own self-harm appeared to have been established before admission to inpatient services. Participants described different levels of preadmission exposure to self-harm that influenced the function and meaning of their own 
self-harm, including self-harm exposure through family, peers and social media. First, participants shared their experiences of familial self-harm. These early and intimate exposure experiences seemed to carry a greater level of power and influence over the young people's relationship with self-harm: “One member of my family used to selfharm so that kind of took an effect. It was hard to see them struggling, so, I guess it just rubbed off” (Sarah). "I've seen my mum self-harm when I was younger... she tried to slit her wrists and it was quite disturbing for me. When I first self-harmed, I hurt myself on my wrists too. I think I got that from seeing her do it” (Joey). Young people found this level of exposure particularly difficult and distressing; perhaps due to the closeness of their relationship with the self-harmer.

The second level of exposure described was to peer self-harm, which for some young people represented their first exposure: "I knew a girl who self-harmed. I used to support her, but people can learn from it and copy it" (Mark); “I didn’t really know what cutting was, but in year eight, it became a phase for a lot of people to do that... so I kind of picked that up" (Helen). However, for those already exposed to their parents' self-harm, these cumulative experiences of exposure may act to normalise and escalate their own self-harm; “There's only so many times you can watch people self-harm around you, like your family and friends, before you start to think its normal and do it yourself" (Joey). When talking about peer exposure, participants did not make reference to the same underlying emotional struggle they did when reflecting on the self-harm of 
their relatives, suggesting that the emotional saliency of the exposure was associated with the type and quality of the young person's relationship with the self-harmer.

The third level of exposure described was via social media. Anna described the internet and social blogging sites as sources of influence over her own and other people's self-harming behaviour; "I had a look on the helpline message boards, and that's how I learnt other ways to do it, which was unhelpful", and Jess expressed her concern that such exposure can promote and glamorise self-harm among young people; "You get so many things on social media and, it just promotes it...in a very sick way it's sort of idolised" (Jess). Exposure to self-harm through social media seemed to serve a different function to exposure through friends and family. Where exposure via friends and family was reported as being associated with the initiation and copying of self-harm as a strategy for managing distress, social media was described here as a means of developing young people's already existing relationship with self-harm (e.g. learning new self-harm methods).

Theme 2: Exposure on the inside: "An unpleasant environment"

When compared with their experiences in the outside world, participants' accounts of exposure on the ward were much more intense and condensed. Being surrounded by the distress of others was difficult for participants; "Most people here were negative about everything and I am a happy person, so that's difficult" (Jack); 
"The fact that someone else is low in mood changes your mood...you can be happy and see someone else struggling makes you struggle" (Sarah). Moreover, Nicola expressed her concern about the consequences of such intense exposure and the impact it would have on the development of her own self-harm:

A lot of people have said hospital is making me worse...it's like you can't win because they bring you in to keep you safe and then you pick up all these things and come out doing more than you did when you came. (Nicola)

Participants described a 'ripple effect' when another young person self-harmed. This effect was described as both physical; "When somebody self-harms it has an effect on everyone else. People's rooms get searched, all the staff are upset...and it has a bad effect on everyone. It makes an unpleasant environment" (Mark); and emotional; “There's always a difficult ward atmosphere... when somebody does something, like self-harms publically... it can set everybody off. It's a difficult place to be" (Jess).

Participants described the ward as having a direct detrimental effect on their well-being; “Sometimes it's traumatising cos you're constantly surrounded by people who self-harm" (Mark); including their own self-harm; "On a basic level, it can just be upsetting to see, but it can also be a negative reminder of the things you want to do yourself" (Jess). In this way, the intensity of exposure to self-harm within inpatient 
settings was socialising young people to self-harm as a means of coping with distress; thus increasing their own risk of self-harm.

In addition to the emotional intensity described, the social dimension of selfharm was also intensified by the physical proximity and undiluted contact with others with similar difficulties. In response to the increased physical restrictions placed upon young people within inpatient settings to keep them safe, such as confiscation of selfharm contraband, participants discussed their exposure to new, and almost 'expert,' selfharm methods:

People get clever with self-harm, you learn places where you can hide things and get things you shouldn't have, you learn how to make everyday objects into something you can harm yourself with... and you learn without trying to. (Jess)

Moreover, participants shared their experiences of how the intense ward environment manifests a social struggle with self-harm; "Three people that hung around together self-harmed on the same night...it was like a chain...one person self-harmed, then another, and another" (Joey); "I see people trying to copy it...when someone's done it [self-harm] then the other person will automatically just go and, it just seems a bit bizarre so close to each other...it just seems so sudden for it to happen in time with each other" (Anna). Seemingly, the intense exposure to the self-harm of others not only 
normalised self-harm but also appeared to contribute towards its transmission in ways that suggested both conscious and unconscious processes at work.

\section{Theme 3: Helper vs Helped}

Despite being admitted to inpatient services to be 'helped', the young people's accounts suggested that exposure to other young people in difficulty often led to them finding themselves in the role of 'helper'; "If you are struggling and someone else is going through pretty much similar you can talk to each other about it and that helps them...you're giving them something positive" (Helen). Helen's quote suggests she valued her role as a helper, perhaps gaining some therapeutic benefit from talking about shared experiences. However, this role could also be emotionally challenging, particularly where it involved being put in a position of having to respond to the selfharm of others. Participants described feeling responsible for the safety of their peers; "Someone yesterday self-harmed and they showed me it...and I didn't think that was right, that's like putting responsibility on me to like basically keep someone safe when I can't keep myself safe" (Joey). Joey felt that, as vulnerable young people, they were being expected to take on a role that they were not equipped for, and that could threaten their own wellbeing. Sarah articulates this even more incisively as a moral dilemma with stark consequences: 
All it takes is one cut in the wrong place and it's all over, so it's kind of scary and worrying and if no one else knows, it puts the pressure on you. You're stuck. Tell and your friend will be mad at you, don't tell and put their life at risk. (Sarah)

In this quote we can see Sarah standing aside from her own emotional vulnerability, her concern is not with her own wellbeing, but with that of her friend. There is also a sense of tension in her helping role in this moment; between providing mutual support to a peer and a more formal helping role aligned with the interests and responsibilities of staff.

Participants also described the negative affect they experienced as a result of not being able to help or prevent others' self-harm; “The fact that we're all in similar ages, you do get a bond and when you see that person get upset it just reminds you of friends struggling. Like how can I help? Why do I feel so worthless?” (Sarah). Here, in a series of short steps, Sarah connects the pressures of the ward environment first to her sense of responsibility for others, and then, tellingly, to her own underlying feelings of worthlessness. Implicit in her quote is an identification of herself with others; the reminder of friends struggling seems to be as much a reminder of her own struggles as those of others. In following the question 'how can I help?' immediately with the question 'why do I feel so worthless?' Sarah makes an implicit connection between her 
sense of helplessness in the face of emotional distress, not only other people's but her own, and her feelings of worthlessness. The ward environment, and the constant exposure to the distress of others serves to reinforce this connection, a connection also articulated by Nicola:

You come in with your own problems and then when you're in here you pick up everyone else's problems and you start thinking about them...it can make you worse and then you feel the need to hate yourself even more. (Nicola)

Another aspect of the role of 'helper' reported by participants was that of young people actively assisting their peers on the ward to self-harm. Here, however, the young people spoke not as active agents but as witnesses of the actions of others, distancing themselves from behaviour which they viewed as morally questionable; "You are meant to be getting better, but to have people who are like helping you do things that you shouldn't do, it has a massive effect” (Jess); “A girl gave another girl a blade because she thought she was being helpful" (Joey); "I think people who self-harm help and promote other people who self-harm. They understand each other and they understand what they're doing" (Jack).

This theme describes how, in the intense environment of the inpatient ward, young people can find themselves taking on the role of 'helper', particularly in relation to the self-harm of others. The role is one which brings with it a range of moral and 
emotional complexities, and which was largely experienced as being detrimental to the recovery of young people who were already struggling with their own mental health.

\section{Theme 4: Separation from the 'attention seekers': competing for authenticity}

This theme captures the complexity of interpersonal relationships amongst peers, both within and outside of the inpatient setting. Participants described their experiences of others making social comparisons; evaluating themselves with reference to their perceptions of others, and then modifying their behaviour to conform to social norms. Such comparisons enabled young people to establish their need for care as authentic; with a distinction drawn between those who self-harmed for attention and those whose self-harm was 'genuine'. This enabled them to make sense of why they needed support from inpatient services, when their self-harming peers in the community did not; "they can just stop [self-harming], but I always found that was something I couldn't do" (Helen). This distinction was also applied to the inpatient setting: "I can tell the difference when people are struggling on the ward and when people are asking for attention" (Nicola).

What emerged from the young people's accounts were reports that aligned themselves with the 'genuine' group, and separated themselves from the socially derided 'attention' group. In aligning themselves with the 'genuine' group, participants benchmarked their self-harm against that of others they identified as genuine, which 
sometimes involved assessing their own level of need as less than others'; "I've met people in a much worse situation than me and it made me feel bad cos the reasons why I self-harm is nowhere near as bad as some people" (Mark); "I sometimes feel a bit pathetic cos mine's not as severe as theirs" (Anna). Jack also benchmarked his own illhealth against his peers' and felt that he had a greater justification to self-harm than most but chose not to; "It surprises me that people want to self-harm...I thought about it, but that's only because I was seriously unwell whereas most people here are fit and healthy" (Jack).

Within the inpatient setting, a sense of competition was described amongst their self-harming peers to use their self-harm as a display of 'genuine' distress and ill health; “It's almost an internal competition really. It's to do with who's the ill-est, who's the worst?" (Helen). As a result, when one young person self-harmed, it appeared to initiate a social reaction among their peers, similar to that described in the first theme; "I see people trying to copy it... sounds really stupid but it's kind of like a competition" (Anna); "Sometimes people think she's getting more attention than me and I'm struggling more so they do it to get attention off other people" (Nicola).

These social comparison processes and self-harm groups were not unique to inpatient contexts; "People like at school who seem to start self-harming and are completely aware of it and the effects that it's gunna have... whereas some people genuinely do it cos they need to cope" (Jess). Jess suggested that the increase in self- 
harming behaviour seen among young people represented a 'trend' whereby young people self-harm for popularity and attention:

I think like self-harming like is becoming like an increasing issue.... I feel like it's sort of become a trend for some people, and you can always tell. I think you can convince yourself that you have a mental illness... and that's what a lot of people do these days. (Jess)

Joey also elaborated on the idea of self-harm becoming more 'trendy' among young people and shared an anecdote about how young people actively attempt to be admitted to inpatient services as validation and a 'statement' of their belonging to the 'genuine' self-harm group:

A person who's been admitted here recently text other people on the ward before to ask how they could get admitted here...most people in here like actually need it and some people get admitted for the whole kind of statement. (Joey)

Joey's quote suggests that young people admitted to inpatient services are perceived as favourable and 'genuine' by their peers in the community.

Another marker which distinguishes between these two groups is the degree to which a young person's self-harm is concealed. In this way, participants highlighted secrecy as a key characteristic of a genuine self-harmer: 
People come up to me and say oh look at me, look what I've done and when you're in a state like that you tend to hide things away from other people and not let them see that you are struggling. (Nicola)

Purposeful displays of self-harm wounds were seen by the young people as a central attribute of the 'attention seeker'; "People would self-harm and walk around wearing short sleeved t-shirts to like show it off' (Jess). Moreover, it appeared that group membership was also determined by the amount of damage inflicted whilst selfharming. Helen identified less severe self-harm as a mark of an attention seeker:

It became more of a fashion statement. It wouldn't be cutting so much as scratching like, they were very very superficial... they don't really struggle, they get a bit sad and they think that self-harm makes them more interesting... they're the kind of people that don't do it to cause much damage. (Helen).

Participants shared their concerns that they would be grouped with other young people who self-harmed for attention by those who did not self-harm themselves; "I was always worried at school that people would think I was part of that so called trend and that people don't take you seriously" (Jess). Being categorised as a self-harmer made them worry that their self-harm would be devalued by the self-harm of those who did it for attention: 
I remember going to hospital to get stitches and I remember thinking they're just gunna think 'oh another teenager self-harming', cos there are so many, I thought it devalued me in a way and made me just one of them. (Helen)

This theme captures how social comparison processes divided young people's perceptions of their peers' self-harm across both community and inpatient settings. Although these groups did not originate within inpatient settings, the need for young people to be recognised by their peers as a member of the socially desirable 'genuine' group and separate from the less favourable 'attention-seeking' group seems to be amplified in such settings. Perhaps more importantly, however, these social comparison processes reflect a struggle to be perceived by others as authentic, leading to an internal conflict between not wanting to be classed as an 'attention seeker' and needing to elicit care from others at times of distress. Social comparison processes exacerbated this conflict and made it difficult for young people to prioritise their own recovery over their need to conform to social norms.

\section{Discussion}

What emerged from the participants' accounts was a profoundly social dimension to both the initiation and maintenance of self-harm in adolescence. Most explanations for self-harm have considered emotional factors such as the alleviation of 
distress and/or infliction of pain as a display of self-hatred (Nock, 2008), however, the present data highlight the presence of significant social influences. The social transmission literature suggests that self-harm is a behaviour susceptible to familial and peer influence (Taiminen et al., 1998), and the participant accounts support this. Participants described different levels of pre-admission exposure that influenced the function and meaning of their own relationship with self-harm. Consistent with previous research (Hasking et al., 2013; Madge et al., 2011), the type and quality of the young person's relationship with the self-harmer seemed to moderate the emotional saliency of the exposure; with intimate relationships being the most influential determinant of their own self-harm.

The exposure environment also appeared to moderate the saliency and influence of the self-harm of others. When compared with their experiences in the outside world, participants' accounts of exposure on the ward were much more intense. All of the young people interviewed found exposure to the distress of others on the ward, and the 'ripple effect' of change that occurred following a young person's self-harm, extremely challenging. Similar experiences were reported by Taiminen et al. (1998), who suggested that the clustering together of young people of similar age, gender and mental health difficulties exacerbated their vulnerability to social transmission and the promotion of self-harming. Moreover, the young people in our study talked about self- 
harm being copied and learnt as a result of their close proximity to exposure on the ward.

Feeling responsible for the safety of peers who self-harm was another social phenomenon to emerge from the participants' accounts. Exposure to other young people in difficulty led to feelings of helplessness and inadequacy when they were not able to help. Since self-harm is thought of as a strategy to cope with intense emotion, an increased number of environmental stressors, may well prove a tipping point between exposure to the self-harm of others and engagement in their own self-harm (Hankin \& Abela, 2011). Therefore, it is crucial that both the physical setting and the peer relationships developed within inpatient services are given careful consideration to ensure that they are helpful and not damaging.

Two distinct groups of self-harmers emerged from the young people's accounts; those who were perceived to self-harm for attention and those perceived as genuine, highlighting further complexity in the interpersonal relationships between young people. A similar divide between groups of self-harmers was also described by participants in Crouch and Wright's (2004) study. Also corresponding to Crouch and Wright's findings, no participant within the present study identified as a member of the 'attention-seeking' group. Of course it is possible that no-one from the attention group was interviewed, however it seems more likely that no young person would purposefully identify themselves as someone who self-harms for attention. 
Participants across both the present study and Crouch and Wright's study were explicit in their concerns that their self-harm would be devalued by the self-harm of those who did it for attention. Secrecy and severity of self-harm were highlighted as key characteristics of the 'genuine' self-harmer, which was particularly concerning given that all the participants who self-harmed identified themselves with this group. The implication was that young people were not only more likely to engage in more severe behaviour to pledge their allegiance to the "genuine self-harmer group", but also do so more privately; significantly inflating their level of risk. Similar findings have been reported by Taiminen et al. (1998) and by Nock (2008), who suggested that the act of self-harm functions as a 'rite of togetherness' or providing a 'sense of belonging' for young people engaging in these behaviours.

As already noted, there are clear correspondences between these findings and those of Crouch and Wright (2004), particularly in young people's identification of two self-harm groups, and the distancing of themselves from the 'attention' group. Crouch and Wright draw on the earlier work of Taiminen (Taiminen, 1992; Taiminen et al., 1998) to develop Klein's idea of projective identification (Klein, 1946) as a possible explanation for this aspect of the social transmission of self-harm. Projective identification is the idea that unwanted feelings or aspects of the self are unconsciously projected onto an external object. Rather than understanding the group dynamics they observed in terms of copying or identification, Crouch and Wright (2004) posit that 
young people unconsciously project the unwanted aspects of themselves onto the 'attention seekers' group, thus freeing themselves temporarily from their own conflicting feelings. The findings from the current study lend further weight to this interpretation of these group dynamics. Not only do our findings provide evidence for the process of projection onto this external entity that, in fact, may not exist except in the minds of those who perceive it to exist (for all participants identified themselves with the genuine group); but they also provide some insight into the unbearable feelings of conflict and turmoil driving this process.

\section{Clinical implications}

The findings reported here strongly support the need for service development to manage the social impact of self-harm within inpatient services, and consider how prevention efforts might help to reduce the social transmission of self-harm. Across accounts, participants noted the benefits of peer support for their self-harm. Participants described a sense of belonging and of feeling understood by other young people with similar struggles and histories, and found the opportunity to share their problems with their peers helpful. However, consideration needs to be given to how this can be managed safely and without the adverse implications of feeling responsible and burdened by the distress of others. 
Research has shown that social support can be protective against self-harm (Hasking et al., 2013). However, the fear of social transmission around this behaviour has led to avoidance of open discussion on this topic (Hasking et al., 2013; McAllister et al. 2010). The introduction of a clinically facilitated self-harm support group within services to encourage a therapeutic milieu (Jones, 2013) may represent a helpful alternative to avoidance of the topic. It would also allow for the sharing of stories to be better controlled and monitored by clinical staff and done in a way which is less detrimental to young people's well-being. Although speculative, fostering a circle of support around the young people (Ward, 2003), which extends beyond professionals, may help to reduce the stigma of being an 'attention seeker' and help towards reducing the social transmission of this behaviour. Indeed, it has been suggested by others that when self-harm is discussed in a neutral way, the frequency of self-harm decreased (Ross \& Mckay, 1979; Taiminen et al., 1998).

Exposure to the self-harm of others is in itself a traumatic experience for young people. In addition to a peer support group, it is crucial that inpatient staff recognise the impact that such exposure has upon the well-being of other young people on the ward and the increased risk of further self-harm incidents. There is a need for staff training on how to manage young people's reactions to the self-harm of others after exposure, and how to work towards creating a more therapeutic environment within inpatient services where young people do not need to engage in competition for care. Moreover, if the 
maintenance and spread of self-harm within inpatient units can be explained, at least in part, by the apparent motivation amongst young people to be recognised as 'authentic' and not an 'attention seeker', it would be helpful for staff to challenge the popular discourse that 'young people harm themselves for attention', which only contributes to the maintenance of such sub-groups (Crouch \& Wright, 2004).

Study limitations and future research

Participation in this study was limited to those proficient in English and the findings of this study may not apply to young people who self-harm but who have not yet come to the attention of services. Recruitment of participants across the two inpatient sites for the present study was also unbalanced, thus the interview conducted at the second service may not have been representative of other young people's experiences of that ward. Nonetheless, the findings do contribute to our understanding of the experiences of young people in inpatient services.

It would be beneficial for researchers to reproduce the current study with participants from non-English speaking backgrounds, and with young people exposed to the self-harm of others in the community. Further qualitative exploration of young people's experiences of the self-harm of others, prior to being admitted to inpatient 
services, would determine whether their experiences and 'sense making' is qualitatively different from young people admitted to inpatient services.

\section{Conclusion}

Despite an abundance of quantitative evidence investigating self-harm in adolescence, there is limited published qualitative research concerning young people's experiences of exposure to the self-harm of others. This study provides pertinent information on the experiences of young people residing within inpatient settings, how young people make sense of the self-harming behaviour of others and the impact of such exposure upon their own well-being. Moreover, this research builds upon existing qualitative studies (Crouch \& Wright, 2004; Taiminen et al., 1998) by providing a more up-to-date account of young people's experiences of the self-harm of others, a broader focus on young people's experiences of self-harm exposure pre-and post-admission, and an understanding of the how social transmission effects and social comparison processes exist amongst young people in inpatient settings. The emotional reactions of the young people towards those who they deem as being an 'attention seeker' may have implications for service delivery, and how services can best support young people to elicit care without being derided for being an 'attention seeker'. 


\section{References}

Brunner, R., Kaess, M., Parzer, P., Fischer, G., Carli, V. et al. (2014). Life-time prevalence and psychosocial correlates of adolescent direct self-injurious behavior: A comparative study of findings in 11 European countries. Journal of Child Psychology and Psychiatry, 55, 337-348. doi:10.1111/jcpp.12166.

Claes, L., Houben, A., Vandereycken, W., Bijttebier, P., \& Mueh- lenkamp, J. (2010). Brief report: The association between non-suicidal self-injury, self-concept and acquaintance with self-injurious peers in a sample of adolescents. Journal of Adolescence, 33, 775-778. doi.org/10.1016/j.adolescence.2009.10.012

Crouch, W., \& Wright, J. (2004). Deliberate self-harm at an adolescent unit: A qualitative investigation. Clinical Child Psychology and Psychiatry, 9: 185-204. doi:10.1177/1359104504041918.

De Leo, D., \& Heller, T. (2004). Who are the kids who self-harm? An Australian selfreport school survey. Medical Journal of Australia, 181: 140-44. 
Deliberto, T., \& Nock, M. (2008). Exploratory study of the correlates, onset, and offset of non-suicidal self-injury. Archives of Suicide Research, 12, 219-231. doi.org/10.1080/13811110802101096

Hankin, B., \& Abela, J. (2011). Nonsuicidal self-injury in adolescence: Prospective rates and risk factors in a 2 1/2 year longitudinal study. Psychiatry Research, 186: 65-70. http://dx.doi.org/10.1016/j.psychres.2010.07.056

Hasking, P., Andrews, T., Graham, M. (2013). The role of exposure to self-injury among peers in predicting later self-injury. Journal of Youth and Adolescence, 42:1543-1556. http://dx.doi.org/10.1007/s10964-013-9931-7

Haw, C., Hawton, K., Niedzwiedz C., \& Platt, S. (2013). Suicide clusters: A review of risk factors and mechanisms. Suicide and Life-Threatening Behavior, 43, 97 108. doi:10.1111/j.1943-278X.2012.00131.x

Hawton, K., Rodham, K., Evans, E., \& Weatherall, R. (2002). Deliberate self-harm in adolescents: self-report survey in schools in England. British Medical Journal; 325: 1207-11. http://dx.doi.org/10.1136/bmj.325.7374.1207

Hawton, K., Saunders, K., \& O'Connor, R. (2012). Self-harm and suicide in adolescents. The Lancet, 379(9834), 2373-2382. doi.org/10.1016/s0140$6736(12) 60322-5$ 
Jarvi, S., Jackson, B., Swenson, L., \& Crawford, H. (2013). The impact of social contagion on non-suicidal self-injury: A review of the literature. Archives of Suicide Research, 17: 1-19. http://dx.doi.org/10.1080/13811118.2013.748404

Jones, M. (2013). Social psychiatry: A study of therapeutic communities. Routledge: Oxon.

Klein, M. (1946). Notes on some schizoid mechanisms. International Journal of Psycho-Analysis, 27: 99-110.

Madge, N., Hawton, K., McMahon, E., Corcoran, P., De Leo., D., De Wilde, E., \& Arensman, E. (2011). Psychological characteristics, stressful life events and deliberate self-harm: findings from the Child and Adolescent Self-harm in Europe (CASE) Study. European Child \& Adolescent Psychiatry, 20(10): 499508. http://dx.doi.org/10.1007/s00787-011-0210-4

McAllister, M., Hasking, P., Estefan, A., McClenaghan, K., \& Lowe, J. (2010). A strengths-based group program on self-harm: A feasibility study. Journal of School Nursing, 26: 289-300. http://dx.doi.org/10.1177/1059840510368801

Morey, Y., Mellon, D., Dailami, N., Verne, J., \& Tapp, A. (2016). Adolescent self-harm in the community: an update on prevalence using a self-report survey of 
adolescents aged 13-18 in England. Journal of Public Health. doi: 10.1093/pubmed/fdw010

Muehlenkamp, J., Brausch, A., Quigley, K., \& Whitlock, J. (2013). Interpersonal features and functions of non-suicidal self-injury. Suicide and Life-Threatening Behavior, 43: 67-80. http://dx.doi.org/10.1111/j.1943-278x.2012.00128.x

Muehlenkamp, J., Claes, L., Havertape, L., \& Plener, P. (2012). International prevalence of adolescent non-suicidal self-injury and deliberate self-harm. Child and Adolescent Psychiatry and Mental Health, 6(1), 1-9. doi: 10.1186/17532000-6-10

National Institute for Health and Clinical Excellence (2011). Self-harm: longer term management. NICE clinical guideline 133. National Collaborating Centre for Mental Health: London.

Nock, M. (2008). Actions speak louder than words: An elaborated theoretical model of the social functions of self-injury and other harmful behaviors. Applied and Preventative Psychology, 12: 159-168.

http://dx.doi.org/10.1016/j.appsy.2008.05.002 
Nock, M., \& Prinstein, M. (2004). A Functional Approach to the Assessment of SelfMutilative Behavior. Journal of Consulting and Clinical Psychology, 72(5): 885-890. http://dx.doi.org/10.1037/0022-006X.72.5.885

Ougrin, D. (2014). Commentary: Self-harm: a global health priority - reflections on Brunner et al. (2014). Journal of Child Psychology and Psychiatry, 55: 349-351. doi:10.1111/jcpp.12232.

Prinstein, M., Heilbron, N., Guerry, J., Franklin, J., Rancourt, D., Simon, V., \& Spirito, A. (2010). Peer Influence and Nonsuicidal Self Injury: Longitudinal Results in Community and Clinically-Referred Adolescent Samples. Journal of Abnormal Child Psychology, 38(5): 669-682. doi.org/10.1007/s10802-010-9423-0.

Rosen, P., \& Walsh, B. (1989). Patterns of contagion in self-mutilation epidemics. The American journal of psychiatry, 146(5): 656. doi.org/10.1176/ajp.146.5.656.

Ross, R., \& McKay, H. (1979). Self-Mutilation. Toronto: Lexington Books.

Smith, J., Flowers, P., \& Larkin, M. (2009). Interpretative Phenomenological Analysis: Theory, Method and Research. Sage publications: London.

Taiminen, T. J. (1992). Projective identification and suicide contagion. Acta Psychiatrica Scandinavica, 85(6), 449-452. 
Taiminen, T., Kallio-Soukainen, K., Nokso-Koivisto, H., Kaljonen, A., \& Kelenius, H. (1998). Contagion of deliberate self-harm among adolescent inpatients. Journal of the American Academy of Child and Adolescent Psychiatry, 37: 211-217. http://dx.doi.org/10.1097/00004583-199802000-00014

Thomassin, K., Guérin Marion, C., Venasse, M., \& Shaffer, A. (2017). Specific coping strategies moderate the link between emotion expression deficits and nonsuicidal self-injury in an inpatient sample of adolescents. Child and Adolescent Psychiatry and Mental Health, 11(1): 21. doi: 10.1186/s13034-017-0158-3

Ward, A. (2003). Therapeutic communities for children and young people (Vol. 10). Jessica Kingsley Publishers: London. 